\title{
On Robustness of a Sequential Test for Scale Parameter of Gamma and Exponential Distributions
}

\author{
Parameshwar V. Pandit ${ }^{1}$, Nagaraj V. Gudaganavar ${ }^{2}$ \\ ${ }^{1}$ Department of Statistics Bangalore University, Bangalore, India \\ ${ }^{2}$ Department of Statistics Anjuman Arts, Science and Commerce, Dharwad, India \\ E-mail:panditpv12@gmail.com \\ Received May 8, 2010; revised July 30, 2010; accepted August 4, 2010
}

\begin{abstract}
The main aim of the present paper is to study the robustness of the developed sequential probability ratio test (SPRT) for testing the hypothesis about scale parameter of gamma distribution with known shape parameter and exponential distribution with location parameter. The robustness of the SPRT for scale parameter of gamma distribution is studied when the shape parameter has undergone a change. The similar study is conducted for the scale parameter of exponential distribution when the location parameter has undergone a change. The expressions for operating characteristic and average sample number functions are derived. It is found in both the cases that the SPRT is robust only when there is a slight variation in the shape and location parameter in the respective distributions.
\end{abstract}

Keywords: Gamma Distribution, Sequential Probability Ratio Test, Operating Characteristic Function, Average Sample Number Function, Robustness

\section{Introduction}

The robustness of sequential probability ratio test (SPRT) has been studied by several authors for various probability distributions when the distribution under consideration has undergone a change. Barlow and Proschan [1], Harter and Moore [2], Montagne and Singpurwalla [3], and others have studied this problem for various probability models.

In this paper, the problem of testing simple hypothesis against simple alternatives for scale parameter of the gamma distribution assuming shape parameter to be known is considered. The gamma distribution plays important role in many areas of the Statistics including areas of life testing and reliability. It is used to make realistic adjustment to exponential distribution in life-testing situations. The fact that a sum of independent exponentially distributed random variables has a gamma distribution, leads to the appearance of gamma distribution in the theory of random counters and other topics associated with precipitation processes.

In Section 2, we state the problem and develop SPRT for testing of hypothesis giving expressions for Operating Characteristic (OC) and Average Sample Number (ASN) functions. In Section 3, robustness of the devel- oped SPRT with respect to OC functions when the distribution considered here has undergone a change in the shape parameter, has been studied. In Section 4 we have studied the robustness of SPRT for the scale parameter of exponential distribution with respect to $\mathrm{OC}$ function when the location parameter has undergone a change.

\section{Materials and Methods}

\section{The set-up of the problem and SPRT}

Let $X_{1}, X_{2}, \ldots$. be a sequence of random variables from a gamma distribution with scale parameter $\theta(>0)$ and shape parameter $\lambda(>0)$, whose density function is given by

$$
f(x: \theta, \lambda)=\frac{x^{\lambda-1} e^{-x / \theta}}{\theta^{\lambda} \Gamma \lambda}, x>0
$$

where it is assumed that $\lambda$ is known. Suppose we want to test the null hypothesis $\mathrm{H}_{0}: \theta=\theta_{0}$ against the alternative $\mathrm{H}_{1}: \theta=\theta_{1}\left(>\theta_{0}\right)$. For this problem following SPRT is developed.

$$
\text { Let } Z=\operatorname{In} \frac{f\left(x ; \theta_{1}\right)}{f\left(x ; \theta_{0}\right)}=\lambda \operatorname{In}\left(\frac{\theta_{0}}{\theta_{1}}\right)+\left(\frac{1}{\theta_{0}}-\frac{1}{\theta_{1}}\right) x
$$

Two numbers $A$ and $B(0<B<1<A)$ are chosen. At 
nth stage accept $\mathrm{H}_{0}$ if $\sum_{i=1}^{n} z_{i} \leq \mathrm{In} B$, reject $\mathrm{H}_{0}$ if

$\sum_{i=1}^{n} z_{i} \geq \operatorname{In} A$, otherwise, continue sampling by taking (n

$+1)$ th observation. Here $Z_{i}$ is obtained by replacing $X$ by $X_{i}$ in (2). Let $(\alpha, \beta)$ be the desired strength of SPRT, then according Wald [4], A and B are approximately given by $A \approx \frac{1-\beta}{\alpha}, B \approx \frac{\beta}{1-\alpha}$, where $\alpha \in(0,1)$ and $\beta \in(0,1)$.

The Operating Characteristic (OC) function $L(\theta)$ is given by

$$
L(\theta) \frac{A^{h(\theta)}-1}{A^{h(\theta)}-B^{h(\theta)}}
$$

Where $h(\theta)$ is the non-zero solution of

$$
E\left(e^{h(\theta) z}\right)=1
$$

From (1) and (2), since

$$
E\left(e^{h(\theta) z}\right)=\left\{1-h(\theta) \theta\left(\frac{1}{\theta_{0}}-\frac{1}{\theta_{1}}\right)\right\}^{-\lambda}\left(\frac{\theta_{0}}{\theta_{1}}\right)^{h(\theta) \lambda}
$$

we get from (4) that

$$
\theta=\frac{1-\left(\frac{\theta_{0}}{\theta_{1}}\right)^{h(\theta)}}{h(\theta)\left(\frac{1}{\theta_{0}}-\frac{1}{\theta_{1}}\right)}
$$

The Average Sample Number (ASN) function is given by

$$
E(N \mid \theta)=\frac{L(\theta) \ln B+[1-L(\theta)] \ln A}{E(Z)}
$$

Provided $E(Z) \neq 0$, where

$$
E(Z)=\lambda\left[\ln \left(\frac{\theta_{0}}{\theta_{1}}\right)+\theta\left(\frac{1}{\theta_{0}}-\frac{1}{\theta_{1}}\right)\right]
$$

The maximum value of ASN occurs in the neighbourhood of $\theta=\tilde{\theta}$, say where $\tilde{\theta}$ is the solution of $E_{\tilde{\theta}}(Z)=0$ and this value is given by

$$
E_{\tilde{\theta}}(N)=\frac{-\ln A \cdot \ln B}{E_{\tilde{\theta}}\left(Z^{2}\right)}
$$

It is easy to see that

$$
\tilde{\theta}=\frac{\ln \left(\theta_{1} / \theta_{0}\right)}{\left(\frac{1}{\theta_{0}}-\frac{1}{\theta_{1}}\right)}
$$

and

$$
E_{\tilde{\theta}}\left(Z^{2}\right)=\lambda\left\{\ln \left(\frac{\theta_{0}}{\theta_{1}}\right)\right\}^{2}
$$

Table 1 contains the values of $E_{0}(N), E_{1}(N)$ and $E_{\tilde{\theta}}(N)$ for different values of $\alpha, \beta$ and ratios of and ratios of $\theta_{0}$ and $\theta_{1}$.

\section{Results and Discussions}

\subsection{Robustness of the SPRT for Scale Parameter of Gamma Distribution}

Let us suppose that there is misspecification for the shape parameter $\lambda$ in the probability distribution. Then the pdf (1) becomes $f\left(x ; \theta, \lambda^{*}\right)$. To study the robustness of the SPRT developed in Section 2 with respect to OC function, consider $h(\theta)$ as the solution of the equation.

$$
\begin{gathered}
E_{\lambda^{*}}\left(e^{h(\theta) z}\right)=1 \\
\text { i.e. } \int_{0}^{\infty}\left[\frac{f\left(x ; \theta_{1}, \lambda\right)}{f\left(x ; \theta_{0}, \lambda\right)}\right]^{h(\theta)} f\left(x ; \theta, \lambda^{*}\right) d x=1
\end{gathered}
$$

giving

$$
\theta=\frac{1-\left(\frac{\theta_{0}}{\theta_{1}}\right)^{\frac{\lambda}{\lambda^{*}} h(\theta)}}{h(\theta)\left(\frac{1}{\theta_{0}}-\frac{1}{\theta_{1}}\right)}
$$

For different values of $\theta, h(\theta)$ is evaluated and the $\mathrm{OC}$ function is obtained. The robustness of SPRT with respect to ASN function can be studied by replacing the denominator of (7) by

$$
E_{\lambda^{*}}(Z)=\int_{0}^{\infty} z f(x ; \theta, \lambda *) d x=\lambda \operatorname{In}\left(\frac{\theta_{0}}{\theta_{1}}\right)+\left(\frac{1}{\theta_{0}}+\frac{1}{\theta_{1}}\right) \theta \lambda *
$$

We consider the cases $\lambda>\lambda *$ and $\lambda<\lambda *$ to study the robustness of the SPRT. In Table 2, we present the ASN function for the cases $\lambda>\lambda *$ for the SPRT of testing the hypothesis $\mathrm{H}_{0}: \theta=45$ against $\mathrm{H}_{1}: \theta=50$. The values of OC function for the cases $\lambda>\lambda^{*}$ and $\lambda<\lambda^{*}$ respectively are plotted Figure 1 and Figure 2.

\subsection{Robustness of SPRT for Exponential Distribution}

The random variable $X$ is said to follow exponential distribution with location parameter $\theta$ and scale parameter $\lambda$ 
Table 1. ASN values of SPRT for scale parameter of gamma distribution.

\begin{tabular}{|c|c|c|c|c|c|c|c|}
\hline \multicolumn{4}{|c|}{$\lambda=5, \alpha=0.01, \beta=0.01$} & \multicolumn{4}{|c|}{$\lambda=5, \alpha=0.05, \beta=0.05$} \\
\hline$\theta_{0} / \theta_{1}$ & $E_{0}(N)$ & $E_{1}(N)$ & $E_{\theta}(N)$ & $\theta_{0} / \theta_{1}$ & $E_{0}(N)$ & $E_{1}(N)$ & $E_{\theta}(N)$ \\
\hline 0.1 & 0.64 & 0.13 & 0.80 & 0.1 & 0.38 & 0.08 & 0.33 \\
\hline 0.2 & 1.11 & 0.38 & 1.63 & 0.2 & 0.65 & 0.22 & 0.67 \\
\hline 0.3 & 1.79 & 0.80 & 2.91 & 0.3 & 1.05 & 0.47 & 1.20 \\
\hline 0.4 & 2.85 & 1.54 & 5.03 & 0.4 & 1.68 & 0.91 & 2.07 \\
\hline 0.5 & 4.66 & 2.94 & 8.79 & 0.5 & 2.74 & 1.73 & 3.61 \\
\hline 0.6 & 8.13 & 5.78 & 16.18 & 0.6 & 4.78 & 3.40 & 16.64 \\
\hline 0.7 & 15.89 & 12.53 & 33.20 & 0.7 & 19.35 & 17.37 & 13.63 \\
\hline 0.8 & 38.92 & 33.54 & 84.81 & 0.8 & 22.90 & 19.73 & 34.82 \\
\hline 0.9 & 168.02 & 156.62 & 380.42 & 0.9 & 198.87 & 192.16 & 156.20 \\
\hline \multicolumn{4}{|c|}{$\lambda=5, \alpha=0.01, \beta=0.01$} & \multicolumn{4}{|c|}{$\lambda=5, \alpha=0.05, \beta=0.05$} \\
\hline$\theta_{0} / \theta_{1}$ & $E_{0}(N)$ & $E_{1}(N)$ & $E_{\theta}(N)$ & $\theta_{0} / \theta_{1}$ & $E_{0}(N)$ & $E_{1}(N)$ & $E_{\theta}(N)$ \\
\hline 0.1 & 0.41 & 0.12 & 0.51 & 0.1 & 0.60 & 0.09 & 0.51 \\
\hline 0.2 & 0.72 & 0.35 & 1.05 & 0.2 & 1.03 & 0.24 & 1.05 \\
\hline 0.3 & 1.15 & 0.74 & 1.88 & 0.3 & 1.66 & 0.52 & 1.88 \\
\hline 0.4 & 1.84 & 1.43 & 3.24 & 0.4 & 2.64 & 1.00 & 3.24 \\
\hline 0.5 & 3.01 & 2.72 & 5.66 & 0.5 & 4.33 & 1.90 & 5.66 \\
\hline 0.6 & 5.25 & 5.36 & 10.42 & 0.6 & 7.54 & 3.73 & 10.42 \\
\hline 0.7 & 10.27 & 11.62 & 21.38 & 0.7 & 14.74 & 18.10 & 21.38 \\
\hline 0.8 & 25.15 & 31.11 & 54.61 & 0.8 & 36.10 & 21.67 & 54.61 \\
\hline 0.9 & 108.58 & 145.27 & 244.96 & 0.9 & 155.84 & 101.22 & 244.96 \\
\hline
\end{tabular}

Table 2. ASN function for $\lambda<\lambda^{*}\left(\mathrm{H}_{0}: \theta=45, \mathrm{H}_{1}: \theta=50, \alpha=\right.$ $\beta=0.05, \lambda=5$.

\begin{tabular}{ccccccc}
\hline$\theta / \lambda^{*}$ & 5.0 & 5.05 & 5.10 & 5.15 & 5.20 & 5.25 \\
\hline 41.00 & 41.30 & 44.11 & 47.32 & 51.03 & 55.33 & 60.39 \\
42.00 & 48.87 & 52.92 & 57.66 & 63.26 & 69.93 & 77.94 \\
43.00 & 59.62 & 65.72 & 73.02 & 81.81 & 92.38 & 104.97 \\
44.00 & 75.50 & 84.94 & 96.24 & 109.58 & 124.72 & 140.68 \\
45.00 & 99.02 & 112.80 & 128.09 & 143.50 & 156.49 & 163.82 \\
46.00 & 129.74 & 144.44 & 155.87 & 160.87 & 157.93 & 147.84 \\
47.00 & 154.07 & 157.58 & 153.15 & 142.24 & 127.85 & 112.80 \\
48.00 & 149.13 & 137.97 & 123.70 & 109.01 & 95.54 & 83.89 \\
49.00 & 120.96 & 106.59 & 93.41 & 82.03 & 72.48 & 64.57 \\
50.00 & 92.35 & 81.06 & 71.59 & 63.74 & 57.25 & 51.83 \\
51.00 & 71.40 & 63.49 & 56.95 & 51.51 & 46.96 & 43.11 \\
52.00 & 57.12 & 51.57 & 46.94 & 43.03 & 39.70 & 36.85 \\
53.00 & 47.25 & 43.23 & 39.82 & 36.89 & 34.36 & 32.16 \\
54.00 & 40.18 & 37.15 & 34.54 & 32.27 & 30.28 & 28.52 \\
55.00 & 34.91 & 32.55 & 30.49 & 28.68 & 27.06 & 25.62 \\
\hline
\end{tabular}

if its probability density function is given by

$$
f(x ; \theta, \lambda)=\lambda e^{-\lambda(x-\theta)}, x>0, \lambda>0
$$

where it is assumed that $\theta$ is known.

Given a sequence of observations $X_{1}, X_{2}, \ldots$, from (13), we wish to test the null hypothesis $\mathrm{H}_{0}: \lambda=\lambda_{0}$ against the alternative $\mathrm{H}_{1}: \lambda=\lambda_{1}\left(>\lambda_{0}\right)$. We propose the following SPRT.

$$
\text { Let } Z=\operatorname{In}\left\{\frac{f\left(x ; \lambda_{1}\right)}{f\left(x ; \lambda_{0}\right)}\right\}=\operatorname{In}\left(\frac{\lambda_{1}}{\lambda_{0}}\right)-\left(\lambda_{1}-\lambda_{0}\right)(x-\theta)
$$

We choose two numbers $A$ and $B$ such that $0<B<1$ $<A$. At $n^{\text {th }}$ stage, accept $\mathrm{H}_{0}$ if $\sum_{i=1}^{n} z_{i} \leq \operatorname{In} B$, reject $\mathrm{H}_{0}$ if $\sum_{i=1}^{n} Z_{i} \geq \ln A$, otherwise continue sampling by taking $(n+$ $1)^{\text {th }}$ observation. Here $Z_{i}$ is obtained by replacing $x$ by $x_{i}$ in (3.2). If $\alpha$ and $\beta$ are the probabilities of type I and type II errors respectively, then $A \cong \frac{1-\beta}{\alpha}, B \cong \frac{\beta}{1-\alpha}$ The $\mathrm{OC}$ function $L(\lambda)$ is given by 


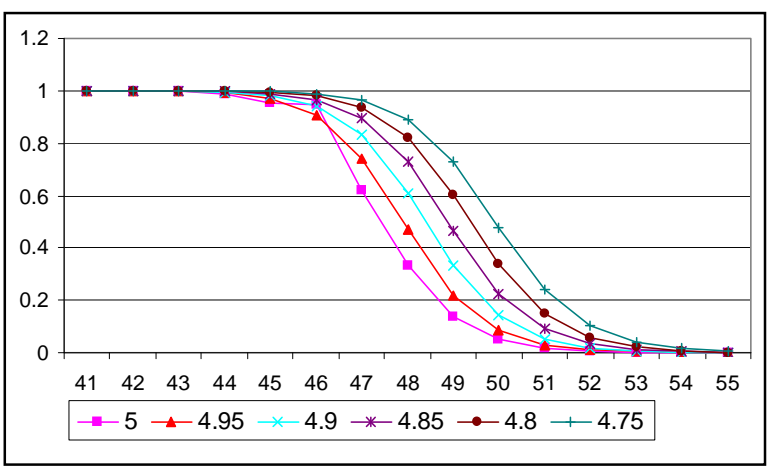

Figure 1. Graph of OC function for gamma distribution $\lambda<$ $\lambda^{*}$.

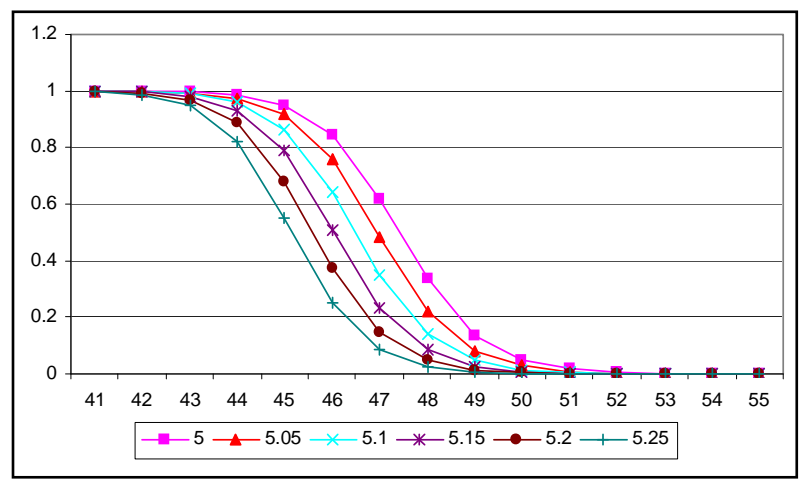

Figure 2. Graph of OC function for gamma distribution $\lambda>$ $\lambda^{*}$.

$$
L(\lambda) \approx \frac{A^{h(\lambda)}-1}{A^{h(\lambda)}-B^{h(\lambda)}},
$$

Where $h(\lambda)$ is the solution of $E\left[e^{h(\lambda) Z}\right]=1$.

Here, $E\left[e^{h(\lambda) Z}\right]=\lambda\left(\frac{\lambda_{1}}{\lambda_{0}}\right)^{h(\lambda)}$ and hence we obtain $\lambda=\left(\frac{\lambda_{0}}{\lambda_{1}}\right)^{h(\lambda)}$.

Let is suppose that the location parameter $\theta$ has undergone a change and the pdf (3.1) becomes $f\left(x ; \theta^{*} ; \lambda\right)$. To study the robustness of the SPRT developed here with respect to OC function, we consider $h(\lambda)$ as the solution of the equation

$$
\int_{0}^{\infty}\left[\frac{f\left(x ; \theta, \lambda_{1}\right)}{f\left(x ; \theta, \lambda_{0}\right)}\right]^{h(\lambda)} f\left(x ; \theta^{*} ; \lambda\right) d x=1
$$

giving

$$
h(\lambda)=\frac{-\ln \lambda}{\left(\lambda_{1}-\lambda_{0}\right)\left(\theta^{*}-\theta\right)+\ln \left(\frac{\lambda_{1}}{\lambda_{0}}\right)}
$$

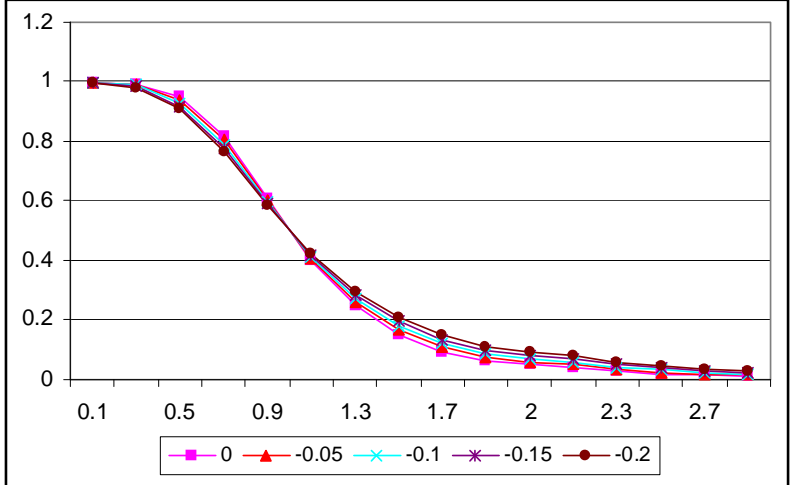

Figure 3. Graph of OC function for exponential distribution $\boldsymbol{\theta}<\boldsymbol{\theta}^{*}$.

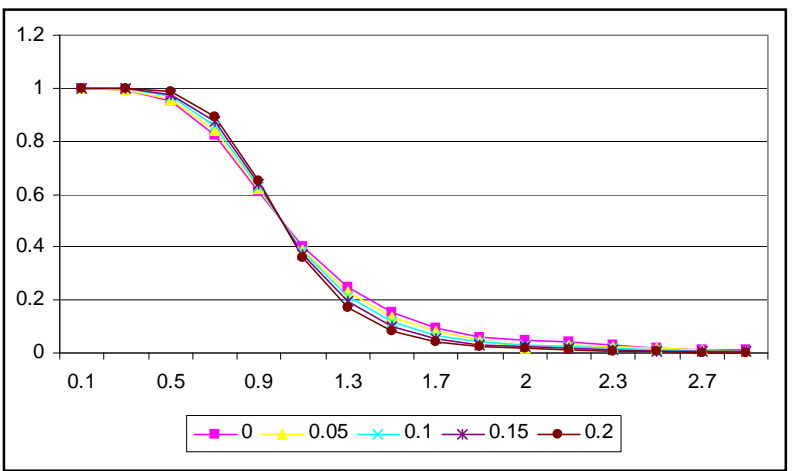

Figure 4. Graph of OC function for exponential distribution $\boldsymbol{\theta}>\boldsymbol{\theta}^{*}$.

For different values of $\lambda, h(\lambda)$ is evaluated and OC function is obtained with the help of Equation (15).

The values of OC function for the cases $\lambda<\lambda^{*}$ and $\lambda>\lambda *$ are plotted in Figures $3 \& \mathbf{4}$ respectively.

\section{Conclusions}

\section{Some Remarks}

1) The values of OC function for the cases $\lambda>\lambda^{*}$ and $\lambda<\lambda^{*}$ respectively are plotted Figure 1 and Figure 2. The figures indicate that for $\lambda>\lambda^{*}\left(\lambda<\lambda^{*}\right)$, the OC curve shifts to the right side (left side) of the curve for when $\lambda=\lambda *$. From the figure it is clear that SPRT is robust for $\lambda^{*}=\lambda \pm 0.05$ as the deviation in OC function is insignificant. However, for other values of $\lambda *$ the test becomes highly non-robust.

2) We have considered the SPRT for testing the hypothesis $\mathrm{H}_{0}: \lambda=1$ versus $\mathrm{H}_{1}: \lambda=2$ for $\alpha=\beta=0.05$. The values of OC function for the cases $\lambda<\lambda^{*}$ and $\lambda>\lambda *$ are plotted in Figures $3 \& 4$ respectively. It is exhibited from the table that the SPRT is non-robust even for $\lambda^{*}=\lambda \pm 0.05$. The ASN function for the case $\lambda<\lambda^{*}$ is tabulated in Table 2 . 


\section{References}

[1] R. E. Barlow and E. Proschan, "Exponential Life Test Procedures When the Distribution under Test has Monotone Failure Rate," Journal of American Statistic Association, Vol. 62, No. 318, 1967, pp. 548-560.

[2] L. Harter and A. H. Moore, "An Evaluation of Exponential and Weibull Test Plans," IEEE Transactions on Re- liability, Vol. 25, No. 2, 1976, pp. 100-104.

[3] E. R. Montagne and N. D. Singpurwalla, "Robustness of Sequential Exponential Life-Testing Procedures," Journal of American Statistic Association, Vol. 80, No. 391, 1985, pp. 715-719.

[4] A. Wald, "Sequential Analysis," John Wiley, New York, 1947. 